\title{
XRF 050406 late-time flattening: an inverse Compton component?
}

\author{
A. Corsi ${ }^{1,2,3}$ and L. Piro ${ }^{1}$ \\ ${ }^{1}$ IASF-Roma/INAF, Via Fosso del Cavaliere 100, 00133 Roma, Italy \\ e-mail: Alessandra.Corsi@iasf-roma.inaf.it \\ 2 Università degli studi di Roma "La Sapienza", Piazzale Aldo Moro 5, 00185 Roma, Italy \\ 3 INFN - Sezione di Roma c/o Dip. di Fisica - Università degli studi di Roma "La Sapienza", Piazzale Aldo Moro 5, \\ 00185 Roma, Italy
}

Received 27 March 2006 / Accepted 19 July 2006

ABSTRACT

\begin{abstract}
Aims. We investigate for possible evidence of inverse Compton (IC) emission in the X-ray afterglow of XRF 050406. Methods. In the framework of the standard fireball model, we show how the late-time flattening observed in the X-ray light curve between $\sim 10^{4} \mathrm{~s}$ and $\sim 10^{6} \mathrm{~s}$ can be explained in a synchrotron-plus-IC scenario when the IC peak frequency crosses the X-ray band. Results. We thus conclude that the appearance of an IC component above the synchrotron one at late times successfully accounts for these X-ray observations.
\end{abstract}

Key words. gamma rays: bursts - X-rays: individuals: XRF 050406 - X-rays: bursts - radiation mechanisms: non-thermal

\section{Introduction}

The "Burst Alert Telescope" (BAT; Barthelmy et al. 2005) on board Swift (Gehrels et al. 2004) was triggered by GRB 050406 on April 6, 2005, at 15:58:48.40 UT (Parsons et al. 2005). The BAT located the burst at RA $=02^{\mathrm{h}} 17^{\mathrm{m}} 53^{\mathrm{s}}$ and $\mathrm{Dec}=$ $-50^{\circ} 10^{\prime} 52^{\prime \prime}$ (J2000), with an uncertainty of 3 arcmin (Krimm et al. 2005). The photon index of the $15-350 \mathrm{keV}$ time-averaged spectrum was $\Gamma=2.38 \pm 0.34$ (Krimm et al. 2005). Because of the spectral softness, this burst was classified as an X-ray flash (XRF; Heise et al. 2001). The $15-350 \mathrm{keV}$ fluence was $\left(1.0_{-0.36}^{+1.13}\right) \times 10^{-7} \mathrm{erg} \mathrm{cm}^{-2}$ (Romano et al. 2006). Assuming a redshift of $z=2.44$ (Schady et al. 2006), the isotropic energy release was $\sim 1.4 \times 10^{51} \mathrm{erg}$.

The "X-Ray Telescope" (XRT; Burrows et al. 2005a) imaged the BAT field starting from $84 \mathrm{~s}$ after the trigger, and the X-ray counterpart of XRF 050406 was found during the on-ground analysis (Cusomano et al. 2005; Capalbi et al. 2005). The "UltraViolet/Optical Telescope" (UVOT; Roming et al. 2005) also started imaging about $88 \mathrm{~s}$ after the trigger. The optical afterglow was not detected on-board (Landsman et al. 2005), but subsequent on-ground analysis revealed a source within the XRT error circle (Rol et al. 2005). Late-time observations ( $\sim .8 \mathrm{~h}$ after the burst) performed by the Magellan/Clay Telescope revealed a single faint source $(R=22.0 \pm 0.09)$ located at $\mathrm{RA}=02^{\mathrm{h}} 17^{\mathrm{m}} 52^{\mathrm{s}} .3$ and Dec $=-50^{\circ} 11^{\prime} 15^{\prime \prime}(\mathrm{J} 2000$; Berger et al. 2005a,b).

The X-ray light curve of XRF 050406 shows some very interesting features. In addition to an X-ray flare (Burrows et al. $2005 \mathrm{~b})$, it is characterized by a flattening at late times $(t \gtrsim 4200 \mathrm{~s}$ from the trigger). In this work we test whether the flattening can be related to the appearance of an IC component (e.g., Wei \& Lu 1998; Panaitescu \& Kumar 2000; Wei \& Lu 2000; Sari \& Esin 2001 ), in the context of the standard fireball model (e.g. Sari et al. 1998). In Sect. 2 we summarize the observed properties of the broad-band afterglow. We refer to the $0.2-10 \mathrm{keV}$ light curve and spectral analysis presented by Romano et al. (2006) and to Table 1 in Schady et al. (2006) for the optical data. In Sect. 3 we constrain the parameters of the fireball in a synchrotron-plus-IC scenario and in Sect. 4 we present our conclusions.

Hereafter, $F(v, t) \propto t^{-\alpha} v^{-\beta}$ is the flux density at the observer's time $t$ and observed frequency $v ; \alpha$ and $\beta$ are the temporal and spectral indices respectively; $E_{52}=E /\left(10^{52} \mathrm{erg}\right)$ is the isotropic equivalent energy of the shock in units of $10^{52} \mathrm{erg}$; $n$ is the particle number density of the ambient medium in units of 1 particle $/ \mathrm{cm}^{3} ; \epsilon_{\mathrm{B},-2}=\epsilon_{\mathrm{B}} / 10^{-2}$ is the fraction of the shock energy, which goes in magnetic energy density behind the shock, in units of $10^{-2} ; \epsilon_{\mathrm{e}, 0.5}=\epsilon_{\mathrm{e}} / 0.5$ is the fraction of shock energy that goes into accelerating the electrons, in units of $0.5 ; p$ is the power-law index of the electron energy distribution (Sari et al. 1998). We adopt $z=2.44$ for the redshift of the source (Schady et al. 2006).

\section{The optical-to-X-ray afterglow}

The $0.2-10 \mathrm{keV}$ light curve of XRF 050406 shows complex behavior (Burrows et al. 2005b), with a power-law decay underlying a flare peaking at about $210 \mathrm{~s}$ after the trigger and a flattening between $\sim 10^{4} \mathrm{~s}$ and $\sim 10^{6} \mathrm{~s}$. Excluding the flare, a broken power-law model with $\alpha_{1}=1.58_{-0.16}^{+0.18}, \alpha_{2}=0.50 \pm 0.14$, and $t_{\text {break }} \sim 4200 \mathrm{~s}$ yields a good fit to the data (Romano et al. 2006).

The mean X-ray energy index was $\beta=1.1 \pm 0.3$ during the first $600 \mathrm{~s}$ after the trigger and $\beta=1.06 \pm 0.24$ between $\sim 600 \mathrm{~s}$ and $\sim 2 \times 10^{4}$ s (Romano et al. 2006). These results were obtained by fitting the data with an absorbed power-law model with hydrogen column density $\left(N_{\mathrm{H}}\right)$ fixed to the Galactic value of $2.8 \times 10^{20} \mathrm{~cm}^{-2}$ (Dickey \& Lockman 1990). The $3 \sigma$ upperlimit for the total (Galactic plus intrinsic) $N_{\mathrm{H}}$ along the line of sight is $9 \times 10^{20} \mathrm{~cm}^{-2}$ (Romano et al. 2006).

The first UVOT observations were performed in the $V$ band, between $113 \mathrm{~s}$ and $173 \mathrm{~s}$. The highest flux was measured in a $50 \mathrm{~s}$ long exposure, starting $113 \mathrm{~s}$ after the trigger. During this observation, the measured magnitude was $18.92 \pm$ 0.31 mag (Schady et al. 2006); after correction for Galactic 
Table 1. Closure relationships between the X-ray afterglow spectral and temporal indices $\left(\beta=1.1 \pm 0.3, \alpha_{1}=1.58 \pm 0.17\right)$ of XRF 050406 in the standard synchrotron fireball model for a wind or ISM environment. In parenthesis: relationships in an ISM modified for the effect of IC cooling. The (u) marks those relationships that are not affected by IC emission.

\begin{tabular}{llcccc}
\hline \hline & & \multicolumn{2}{c}{ ISM environment } & \multicolumn{2}{c}{ Wind environment } \\
\hline & & Expected relation & Observed value & Expected relation & Observed value \\
a) & $v_{\mathrm{c}}<v_{\mathrm{X}}<v_{\mathrm{m}}$ & $2 \alpha-\beta=0(\mathrm{u})$ & $2.1 \pm 0.4$ & $2 \alpha+\beta-1=0$ & $3.3 \pm 0.5$ \\
$\mathrm{~b})$ & $v_{\mathrm{c}}<v_{\mathrm{m}}<v_{\mathrm{X}}$ & $2 \alpha-3 \beta+1=0(\mathrm{u})$ & $0.86 \pm 0.96$ & $2 \alpha-3 \beta+1=0$ & $0.86 \pm 0.96$ \\
c) & $v_{\mathrm{m}}<v_{\mathrm{X}}<v_{\mathrm{c}}$ & $2 \alpha-3 \beta=0(\mathrm{u})$ & $-0.14 \pm 0.96$ & $2 \alpha-3 \beta-1=0$ & $-1.1 \pm 1.0$ \\
d) & $v_{\mathrm{m}}<v_{\mathrm{c}}<v_{\mathrm{X}}$ & $2 \alpha-3 \beta+1=0\left(\alpha+\frac{3 \beta^{2}-6 \beta+1}{2(2-\beta)}=0\right)$ & $0.86 \pm 0.96(0.48 \pm 0.31)$ & $2 \alpha-3 \beta+1=0$ & $0.86 \pm 0.96$ \\
\hline
\end{tabular}

extinction $\left(E(B-V)=0.022 \mathrm{mag}\right.$ and $A_{V} / E(B-V)=3.1$, Schlegel et al. 1998), this corresponds to a $V$-band flux of $0.11 \pm$ $0.04 \mathrm{mJy}$. Since at about $100 \mathrm{~s}$ the $0.2 \mathrm{keV}$ flux was $\sim 0.05 \mathrm{mJy}$ (using a conversion factor of $6.5 \times 10^{-11} \mathrm{erg} \mathrm{cm}^{-2}$ count $^{-1}$ and a spectral index of $\beta \sim 1.1$, as found by Romano et al. 2006), the observed optical-to-X-ray spectral index at $100 \mathrm{~s}, \beta_{\text {opt-X }} \sim 0.15$, turned out to be extremely flat. Such a flat value was not found in the analysis performed by Schady et al. (2006), where the mean optical and X-ray flux between $220 \mathrm{~s}$ and $950 \mathrm{~s}$ were considered.

\section{Synchrotron plus IC model}

As seen in the previous section, the X-ray light curve of XRF 050406 is characterized by a steep decay followed by a flattening after $t \sim 4200 \mathrm{~s}$ (see e.g. Fig. 1). Recently, Chincarini et al. (2005) and Nousek et al. (2006) found that the X-ray light curves of Swift GRBs usually present an initial steep decay ( $t \lesssim 500 \mathrm{~s}$ ), followed by a shallower one and, finally, by further steepening. In the case of XRF 050406, the temporal decay observed before the break $\left(\alpha_{1} \sim 1.58\right)$ is not as steep as usual ( $3 \leq \alpha \leq 5$, Tagliaferri et al. 2005) and the curvature relation $\alpha=\beta+2$ is not satisfied. These arguments suggest that the emission preceding the late-time flattening could be part of the afterglow (Romano et al. 2006) rather than the tail of the prompt emission. In this scenario, the superimposed flare could be interpreted in the framework of the late internal-shock model (Burrows et al. 2005b; Fan \& Wei 2005; Wu et al. 2005; Romano et al. 2006). On the other hand, if the flare marks the onset of the afterglow (Piro et al. 2005; Galli \& Piro 2006), the analysis presented here is valid for $t>210 \mathrm{~s}$.

Several authors have considered the effect of IC emission in GRB X-ray light curves at late times (e.g. Wei \& Lu 2000; Sari \& Esin 2001). In this section we test a model in which the steep part of the X-ray light curve is produced by synchrotron emission while the late-time flattening is due to the appearance of an IC component at that point in time.

\subsection{Synchrotron component}

In Table 1 we summarize the closure relationships between the spectral index $\beta$ and the early temporal decay index $\alpha_{1}$ for the X-ray afterglow of XRF 050406. The relation

$\nu_{\mathrm{m}}=2 \times 10^{13} \frac{f(p)}{f(2.5)}(1+z)^{1 / 2} \epsilon_{\mathrm{B},-2}^{1 / 2} \epsilon_{\mathrm{e}, 0.5}^{2} E_{52}^{1 / 2} t_{\mathrm{d}}^{-3 / 2} \mathrm{~Hz}$

is the injection frequency, with $f(p)=\left(\frac{p-2}{p-1}\right)^{2}$, and

$v_{\mathrm{c}}=2.7 \times 10^{15}(1+z)^{-1 / 2} \epsilon_{\mathrm{B},-2}^{-3 / 2} E_{52}^{-1 / 2} n^{-1} t_{\mathrm{d}}^{-1 / 2}(1+x)^{-2} \mathrm{~Hz}$

is the cooling frequency (Sari \& Esin 2001); $t_{\mathrm{d}}$ is the observer's time in units of days; $x$ is the ratio of the IC to
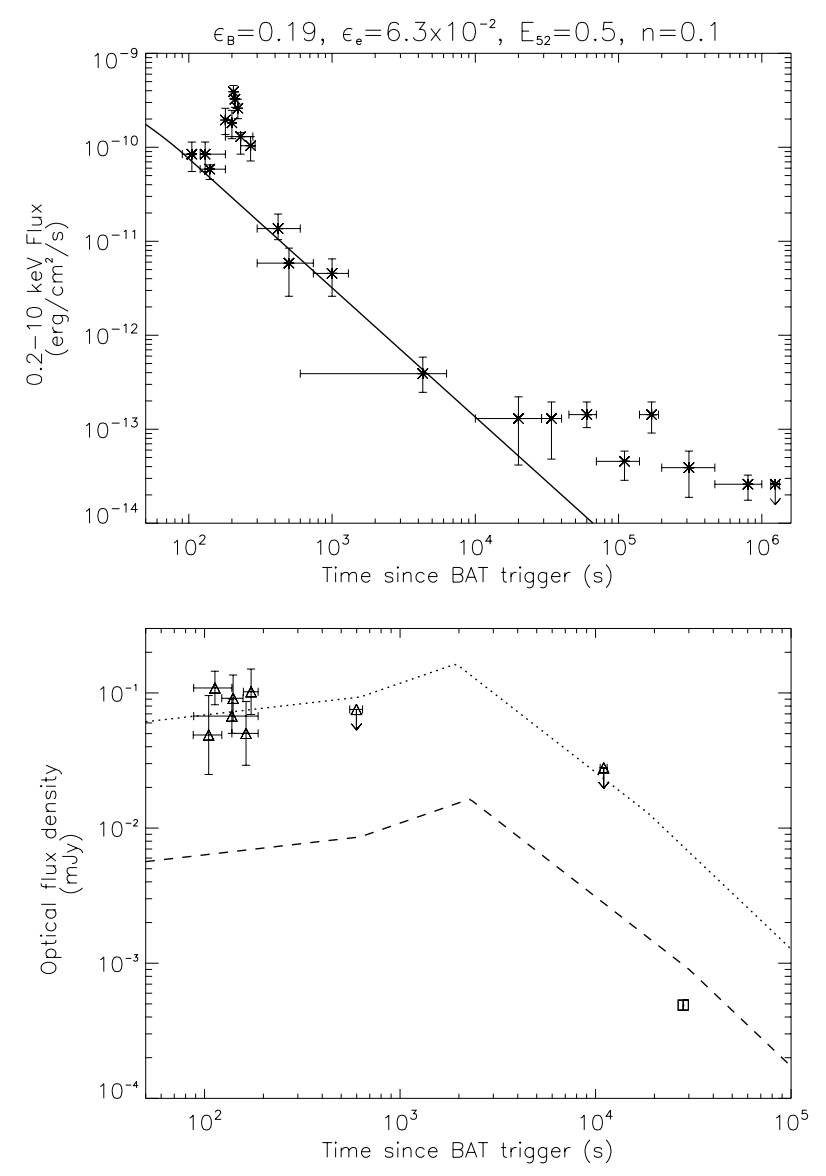

Fig. 1. 0.2-10 keV (upper panel, solid line), $V$-band (lower panel, dotted line), and $R$-band (lower panel, dashed line) light curves in a standard synchrotron fireball model with $\epsilon_{\mathrm{B}}=0.19, \epsilon_{\mathrm{e}}=6.3 \times 10^{-2}$, $E_{52}=0.5, n=0.1, p=2.5$, so as to fit the observed optical and X-ray fluxes at $100 \mathrm{~s}$, i.e. to satisfy the conditions $v_{\mathrm{m}}(100 \mathrm{~s}) \sim 0.2 \mathrm{keV}$, $v_{\mathrm{c}}(100 \mathrm{~s}) \sim 0.03 \mathrm{keV}$ (see text). The $0.2-10 \mathrm{keV}$ data points (upper panel, crosses), the $V$-band data points (lower panel, triangles), and the the $R$-band data point (lower panel, box) are taken from Romano et al. (2006), Schady et al. (2006), and Berger et al. (2005a), respectively. The $R$-band light curve and data point have been shifted down by a factor of 10 for clarity.

synchrotron luminosity, i.e. $x \sim \sqrt{\epsilon_{\mathrm{e}} / \epsilon_{\mathrm{B}}}$ in the fast-cooling regime and $x \sim \sqrt{\epsilon_{\mathrm{e}} / \epsilon_{\mathrm{B}}} \times\left(t / t_{\mathrm{IC}}^{0}\right)^{-(p-2) /(2(4-p))}$ in the slow-cooling IC-dominated regime ${ }^{1}$ (Sari \& Esin 2001); $t_{\mathrm{IC}}^{0}$ is the time at which the transition from slow cooling to fast cooling takes place (when $v_{\mathrm{m}}$ equals $v_{\mathrm{c}}$ ). It is important to note that if IC is an

1 These expressions for $x$ are valid if $x^{2} \gg 1$ (Sari \& Esin 2001). 
efficient cooling mechanism, the transition from fast cooling to slow cooling is delayed by a factor of $\epsilon_{\mathrm{e}} / \epsilon_{\mathrm{B}}$ (Sari \& Esin 2001).

In the case of a constant density interstellar medium (ISM), we indicate in parenthesis the closure relationships modified for the inclusion of IC emission. In particular in scenario d), the same relation of case b) holds, when considering only synchrotron emission. However, the addition of IC modifies the expression of $v_{\mathrm{c}}$ for a factor of $(1+x)^{-2}$. Since during the slow cooling regime, $x$ evolves with time, the temporal decay index at $v \geq v_{\mathrm{c}}$ changes from $3 / 4(p-1)+1 / 4$ to $3 / 4(p-1)+1 / 4-(p-$ $2) /(8-2 p)$ (Sari \& Esin 2001; Corsi et al. 2005) and the corresponding closure relationship changes as indicated in parenthesis in Table 1. In scenario c), since the flux at $v \leq v_{\mathrm{c}}$ does not depend on the expression of $v_{\mathrm{c}}$, the closure relationship is not modified. Finally, in the fast-cooling regime, $x$ does not depend on time, thus the temporal decay index at frequencies above $v_{\mathrm{c}}$ does not change (cases a) and b)).

On the basis of the closure relationships, scenarios b), c), and d) are compatible with the observations in both an ISM and a wind environment (Chevalier \& Li 1999). Hereafter, we will limit our discussion to the ISM case.

We have seen in Sect. 2 that the optical-to-X-ray spectral index before the flare is rather flat. In a standard synchrotron scenario, such a flat value cannot be explained unless the synchrotron peak frequency is between the optical and the X-ray band. In Fig. 1 we show that setting $v_{\mathrm{c}}(100 \mathrm{~s}) \sim 0.03 \mathrm{keV}$ (peak frequency between the optical and the X-ray band) and $v_{\mathrm{m}}(100 \mathrm{~s}) \sim 0.2 \mathrm{keV}$ (case b) in Table 1), marginal consistency with the data can be obtained. However, under these conditions, $v_{\mathrm{m}}$ crosses the $V$-band around $2 \times 10^{3} \mathrm{~s}$; until that time, the light curve (Fig. 1) features a rise, while XFR 050406 was no longer visible at $\sim 600 \mathrm{~s}$ above the background (Schady et al. 2006), suggesting a progressive fading of the optical afterglow (the $V$-band upper-limits adopted here are standard $3 \sigma$ upper-limits, thus the corresponding limiting fluxes are 3 times higher than the $1 \sigma$ upper-limits reported by Schady et al. 2006). Note also that in a synchrotron scenario, $\beta \gtrsim 0.5$ at frequencies above the peak one. Since $v_{\mathrm{c}}(100 \mathrm{~s}) \sim 0.03 \mathrm{keV}$ and $v_{\mathrm{m}}(100 \mathrm{~s}) \sim 0.2 \mathrm{keV}$ imply $\beta=0.5$ for $v_{\mathrm{c}} \leq v \leq v_{\mathrm{m}}=0.2 \mathrm{keV}$, in Fig. 1 we are minimizing the peak-flux value required to fit the optical and X-ray data at $100 \mathrm{~s}$, thus minimizing the rise in the optical flux observed until the peak frequency is above the optical band.

To account for a decreasing optical emission, the synchrotron peak frequency should be below the optical band. In this case, to fit the early X-ray afterglow while overestimating as little as possible the optical flux around $\sim 100 \mathrm{~s}$, the best choice is to set $v_{\mathrm{c}}(100 \mathrm{~s}) \leq 10^{14} \mathrm{~Hz}$ and $v_{\mathrm{m}}(100 \mathrm{~s}) \sim 0.2 \mathrm{keV}$ (case b) in Table 1). In fact, then $\beta_{\text {opt-X }}=0.5$, which is the flattest value allowed in a standard synchrotron scenario when the peak frequency is below the optical band (and $p>2$ ). Under these assumptions, normalizing to the observed X-ray flux at $100 \mathrm{~s}$, the predicted $V$-band flux is overestimated for a factor of $\sim 5$. In the $R$-band, the predicted light curve will decrease as $t^{-1 / 4}$ until the time at which $v_{\mathrm{m}}$ crosses the band $(\sim 2300 \mathrm{~s})$ and as $t^{-\frac{3}{4}(p-1)-\frac{1}{4}}$ up to the time of the Magellan/Clay observation $\left(t \sim 3 \times 10^{4} \mathrm{~s}\right)$. Since in this case the measured X-ray spectral and temporal indices imply $\langle p\rangle=2.5 \pm 0.3$, then $t^{-\frac{3}{4}(p-1)-\frac{1}{4}}=t^{-1.375}$, and we expect the predicted $R$-band flux to be around a factor of $\sim 2$ above the Magellan/Clay data point. Requiring additional extinction in the GRB host galaxy or a contribution to the early X-ray flux coming from the rising part of the flare (or a combination of these two effects) helps account for the broad-band observations, as we see in the following section.

\subsection{IC component}

To model the observed early-time emission within the synchrotron fireball model we choose scenario b) of Table 1 with $v_{\mathrm{c}}(100 \mathrm{~s}) \leq 10^{14} \mathrm{~Hz}$ and $v_{\mathrm{m}}(100 \mathrm{~s}) \sim 0.2 \mathrm{keV}$. We thus set $p=2.5$.

To model the flattening observed in the X-ray afterglow at late times, we add the contribution of an IC component. Following the prescriptions given by Sari \& Esin (2001), the IC spectrum is modeled as a power-law spectrum similar to the synchrotron one, plus logarithmic corrections when relevant in the considered regime. In the power-law approximation, the IC spectrum is normalized to a peak-flux value of $f_{\max }^{\mathrm{IC}}=2 \times 10^{-7} f_{\max } n\left(R / 10^{18}\right)$, where $f_{\max }$ is the peak flux of the synchrotron component and $R$ is the fireball radius in $\mathrm{cm}$ (Sari \& Esin 2001).

To constrain the values of $E_{52}, n, \epsilon_{\mathrm{e}}, \epsilon_{\mathrm{B}}$ that reliably circumscribe the portion of parameter space compatible with the scenario we are testing, we set the following conditions:

$0.1 \mathrm{keV} \lesssim v_{\mathrm{m}}(100 \mathrm{~s}) \lesssim 0.3 \mathrm{keV}$,

$v_{\mathrm{c}}(100 \mathrm{~s}) \lesssim 10^{14} \mathrm{~Hz}$,

$50 \times 10^{-3} \mathrm{mJy} \lesssim f_{0.2 \mathrm{keV}}^{\mathrm{syn}}(100 \mathrm{~s}) \lesssim 70 \times 10^{-3} \mathrm{mJy}$,

where $f_{0.2 \mathrm{keV}}^{\mathrm{syn}}(100 \mathrm{~s})=f_{\max }^{\mathrm{syn}}\left(\frac{0.2 \mathrm{keV}}{v_{\mathrm{c}}(100 \mathrm{~s})}\right)^{-0.5}$ if $v_{\mathrm{m}}(100 \mathrm{~s})>$ $0.2 \mathrm{keV}$ or $f_{0.2 \mathrm{keV}}^{\mathrm{syn}}(100 \mathrm{~s})=f_{\max }^{\mathrm{syn}}\left(\frac{v_{\mathrm{m}}(100 \mathrm{~s})}{\gamma_{\mathrm{c}}(100 \mathrm{~s})}\right)^{-0.5}\left(\frac{0.2 \mathrm{keV}}{v_{\mathrm{m}}(100 \mathrm{~s})}\right)^{-p / 2}$, if $v_{\mathrm{m}}(100 \mathrm{~s})<0.2 \mathrm{keV}$ (depending on Eq. (3) and supposing that Eq. (4) is valid).

Equations (3) and (4) take the requirement to overestimate as little as possible the early-time optical flux into account (see Sect. 3.1) and reproduce the observed X-ray spectral and temporal indices (for $p=2.5$, see Table 1); Eq. (5) is a normalization condition on the X-ray light curve based on the observed $0.2-10 \mathrm{keV}$ flux at $100 \mathrm{~s}$ normalized to a frequency of $10^{18} \mathrm{~Hz}$ (at the center of the X-ray band). Moreover, to relate the observed flattening to the appearance of an IC component, the following conditions have to be satisfied:

$4.5 \times 10^{-6} \mathrm{mJy} \lesssim f_{\max }^{\mathrm{IC}}\left(10^{5} \mathrm{~s}\right) \lesssim 10^{-5} \mathrm{mJy}$,

$v_{\mathrm{m}}^{\mathrm{IC}}\left(10^{5} \mathrm{~s}\right) \gtrsim 5 \mathrm{keV}$,

$v_{\mathrm{m}}^{\mathrm{IC}}\left(10^{6} \mathrm{~s}\right) \lesssim 0.2 \mathrm{keV}$.

Considering that in both the fast and slow cooling regimes the IC light curve at a given frequency is expected to decline only after $v_{\mathrm{m}}^{\mathrm{IC}}$ becomes lower than that frequency, Eqs. (7) and (8) are set to reproduce the observed shape of the late-time flattening. In the $0.2-10 \mathrm{keV}$ band, the IC component should have a constant or rising profile up to $10^{5} \mathrm{~s}$, while after that it should start decreasing, and around $10^{6} \mathrm{~s}$ it should no longer be visible above the background. We numerically tested for which values of $\epsilon_{\mathrm{B}}$ and $\epsilon_{\mathrm{e}}$ the above conditions are simultaneously satisfied, once the values of $E_{52}$ and $n$ are fixed. Figure 2 shows some examples on how the conditions expressed in Eqs. (3) to (8) determine the allowed range of parameter values.

Among the possible combination of parameters satisfying the conditions we set to determine the most promising portion of the parameter space, one should then specifically check for agreement with the actual data. In fact, in Fig. 3 we show how, for $\epsilon_{\mathrm{B}}=1.9 \times 10^{-4}, \epsilon_{\mathrm{e}}=0.25, E_{52}=5, n=100$, the appearance of an IC component can be a viable model to explain the latetime flattening observed in the X-ray light curve of XRF 050406. 

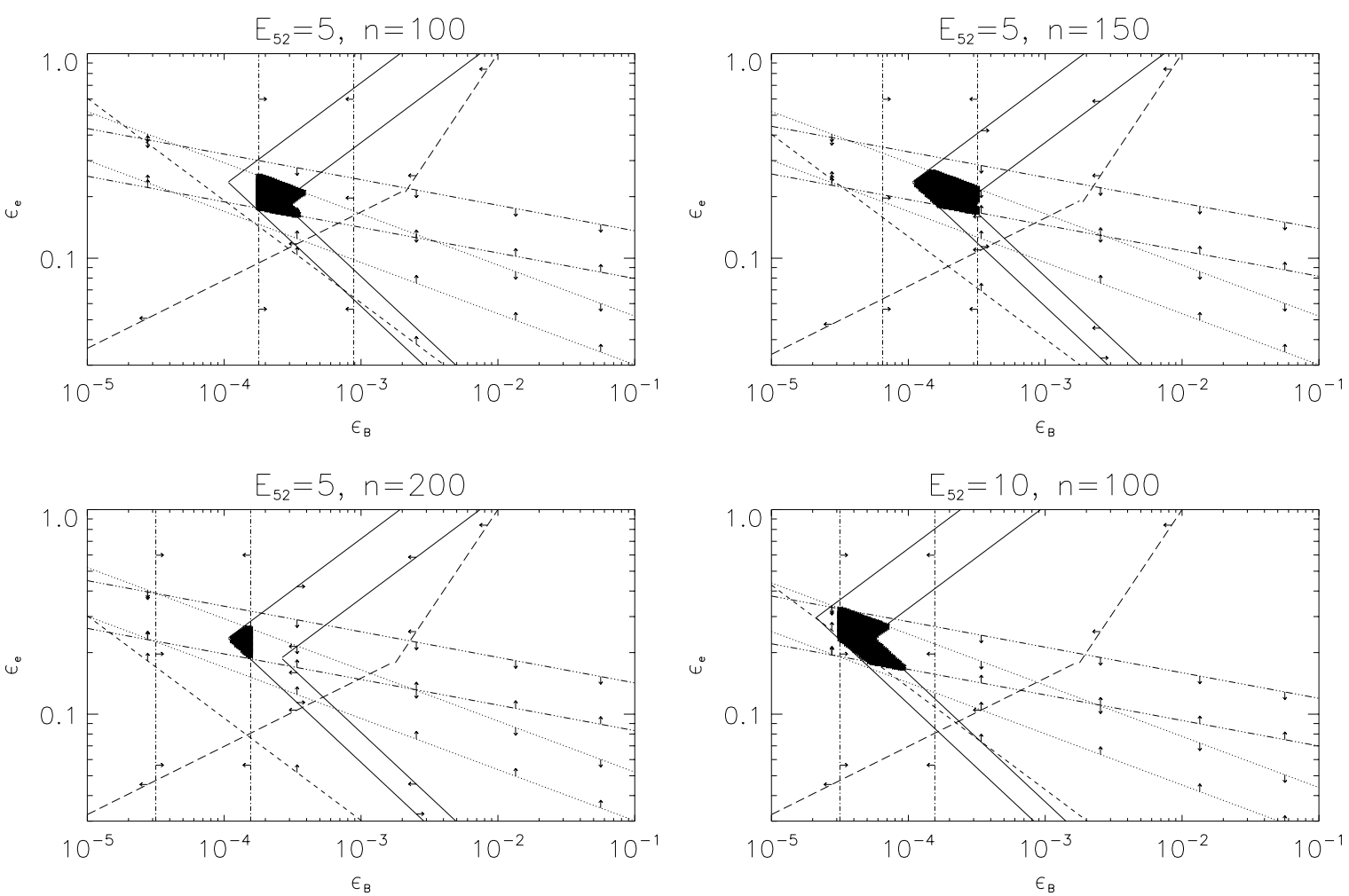

Fig. 2. The shadowed regions are the parts of the $\epsilon_{\mathrm{B}}-\epsilon_{\mathrm{e}}$ plane where the conditions expressed by Eq. (3) (dotted lines), Eq. (4) (dashed line), Eq. (5) (solid lines), Eq. (6) (dash-dotted lines), Eqs. (7) and (8) (dash-dot-dot-dotted lines) are simultaneously satisfied, for $E_{52}=5$ and $n=100$ (upper-left panel), $E_{52}=5$ and $n=150$ (upper-right panel), $E_{52}=5$ and $n=200$ (lower-left panel), and $E_{52}=10$ and $n=100$ (lower-right panel). The long-dashed line marks the portion of the $\epsilon_{\mathrm{B}}-\epsilon_{\mathrm{e}}$ plane where $x=\left(\frac{\epsilon_{\mathrm{e}}}{\epsilon_{\mathrm{B}}}\right)^{1 / 2}>10$ if $t_{0}^{\mathrm{IC}}>10^{6} \mathrm{~s}$ and $x\left(10^{6} \mathrm{~s}\right)=\left(\frac{\epsilon_{\mathrm{e}}}{\epsilon_{\mathrm{B}}}\right)^{1 / 2}\left(\frac{10^{6} \mathrm{~s}}{t_{0}^{\mathrm{IC}}}\right)^{-\frac{(p-2)}{2(4-p)}}>10$ if $t_{0}^{\mathrm{IC}}<10^{6}$, so as to assure that $x>1$ up to $10^{6} \mathrm{~s}$, which is necessary for the consistency of our formulation (Sari \& Esin 2001).

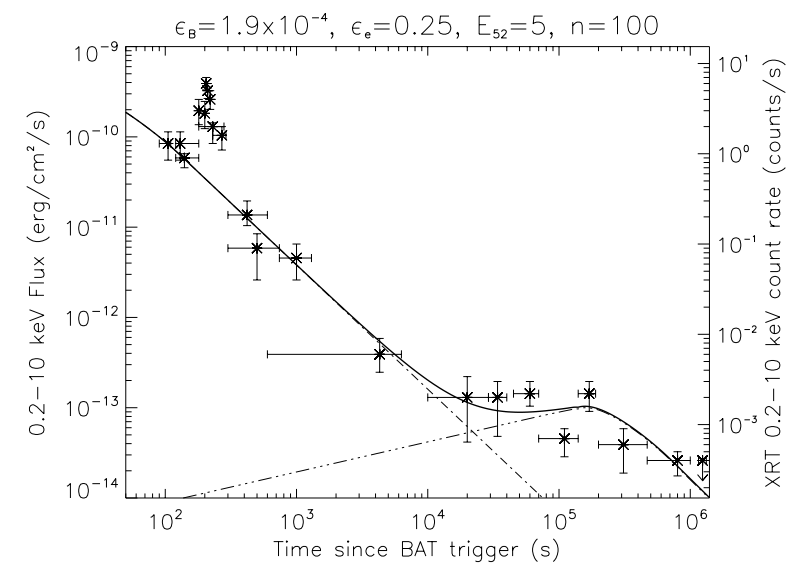

Fig. 3. Synchrotron-plus-IC model predictions for the $0.2-10 \mathrm{keV}$ light curve with $\epsilon_{\mathrm{B}}=1.9 \times 10^{-4}, \epsilon_{\mathrm{e}}=0.25, E_{52}=5, n=100, p=2.5$. The dash-dotted line represents the synchrotron component, while the dashdot-dot-dotted line the IC one. The solid line is the resulting total flux. The model predictions are compared with the $0.2-10 \mathrm{keV}$ data points (crosses) by Romano et al. (2006).

For the same set of parameters, we also computed the predicted $V$ - and $R$-band light curves. As expected (Sect. 3.1), if no local extinction is added, the predicted $V$ - and $R$-band fluxes are overestimated of a factor of $\sim 5$ and $\sim 2.4$, respectively. In a "Small Magellanic Clouds" (SMC)-like environment (Pei 1992), an intrinsic extinction of $A\left(V_{\text {int }}\right) \sim 0.32 \mathrm{mag}$ in the GRB site (at $z=2.44$ ) allows consistency to be recovered with the optical data (Fig. 4, upper panel); this implies an absorption column density of $N_{\mathrm{H}} \sim 0.32 \times 1.6 \times 10^{22} \mathrm{~cm}^{-2}$. In such an environment, due to the lower metallicity, the upperlimit of $9 \times 10^{20} \mathrm{~cm}^{-2}$ set by the XRT analysis should be increased by a factor of $\sim 7$ (Stratta et al. 2004), and thus the required $N_{\mathrm{H}}$ should be compared with this increased limit. The numerical test that guided us in finding this solution was repeated for $E_{52}=0.5,1,2,3,5,10$, combined with $n=$ $10,50,100,150,200,250,300,350,400$. Solutions are found for $E_{52}=3,5,10$ (Fig. 5, right-to-left) and for $n=$ 100, 150, 200.

If before the X-ray flare (say $t \lesssim 300 \mathrm{~s}$ ) there is some contribution from the rising part of the flare itself (as one may expect in a late internal shock scenario), we can relax the normalization condition to some extent. Setting $v_{\mathrm{m}}(300 \mathrm{~s}) \sim 0.2 \mathrm{keV}$ (so as to reproduce the observed X-ray spectral and temporal indices after the flare for $p=2.5$ ) and normalizing the synchrotron spectrum to fit the observed X-ray flux level at $\sim 300 \mathrm{~s}$, the $0.2 \mathrm{keV}$ light curve at $t \lesssim 300 \mathrm{~s}$ will rise slowly (as $t^{-1 / 4}$ ), and the $0.2 \mathrm{keV}$ flux at $100 \mathrm{~s}$ will be lowered by a factor of $\frac{(300 \mathrm{~s} / 100 \mathrm{~s})^{-1 / 4}}{(300 \mathrm{~s} / 100 \mathrm{~s})^{-3(p-1) / 4-1 / 4}} \sim 3$ with respect to the previous solution (Fig. 3). Extrapolating to the optical band, the expected $V$-band flux will overestimate the observed one by a factor of $\sim 5 / 3 \sim 2$, instead of $\sim 5$.

Under this hypothesis, we can shift the time in Eqs. (3) and (4) from $100 \mathrm{~s}$ to $300 \mathrm{~s}$ and modify the normalization condition on the synchrotron component (Eq. (5)) to fit the observed X-ray flux after the flare:

$8 \times 10^{-3} \mathrm{mJy} \lesssim f_{0.2 \mathrm{keV}}^{\mathrm{syn}}(300 \mathrm{~s}) \lesssim 20 \times 10^{-3} \mathrm{mJy}$ 

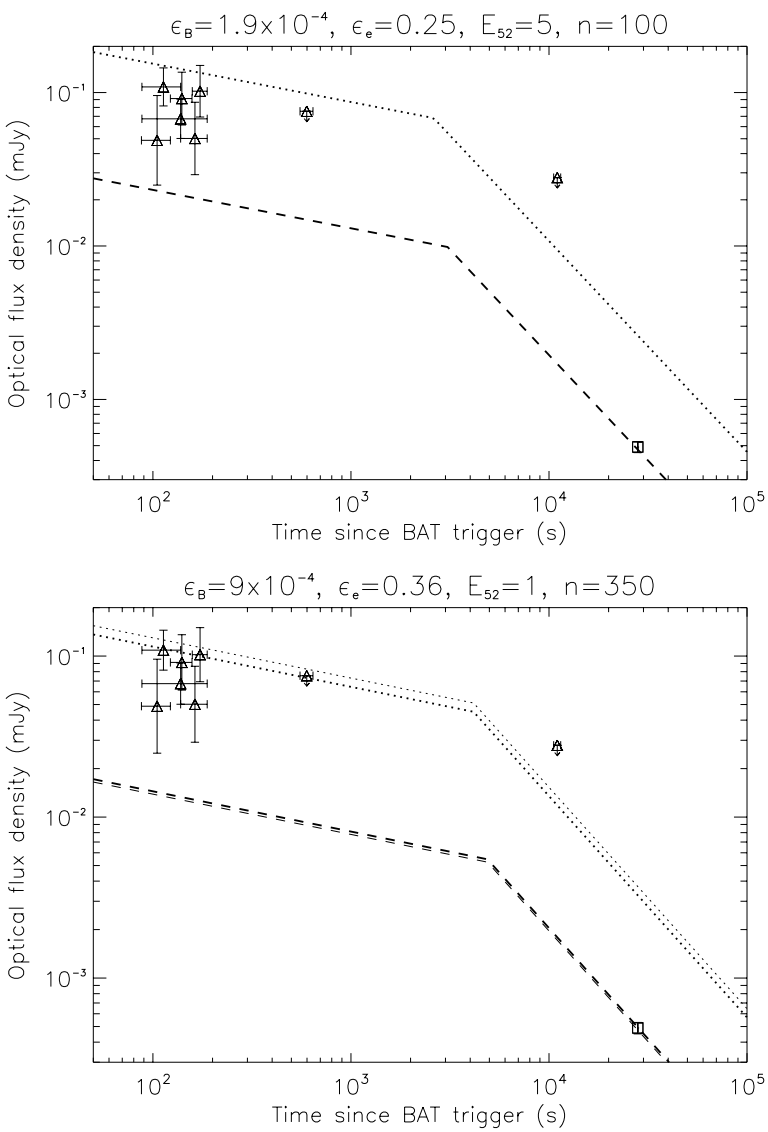

Fig. 4. Synchrotron-plus-IC model predictions compared with the observed data in the case $\epsilon_{\mathrm{B}}=1.9 \times 10^{-4}, \epsilon_{\mathrm{e}}=0.25, E_{52}=5, n=100$, $p=2.5$ (upper panel) and $\epsilon_{\mathrm{B}}=9 \times 10^{-4}, \epsilon_{\mathrm{e}}=0.36, E_{52}=1$, $n=350, p=2.5$ (lower panel). In the upper panel, the $V$-band (dotted line) and $R$-band (dashed line) light curves include a SMC-like local $(z=2.44)$ extinction of $A\left(V_{\text {int }}\right) \sim 0.32 \mathrm{mag}$; in the lower panel, the $V$-band (dotted lines) and $R$-band (dashed lines) light curves include an SMC-like extinction of $A\left(V_{\text {int }}\right) \sim 0.13$ mag (thick lines) or a Galacticlike $A\left(V_{\text {int }}\right) \sim 0.15 \mathrm{mag}$ (thin lines). In both panels the $R$-band light curve and data point have been shifted down by a factor of 10 for clarity.

Similar to what done before, we numerically searched for the values of $\epsilon_{\mathrm{B}}$ and $\epsilon_{\mathrm{e}}$ that allow us to satisfy simultaneously those conditions, for a given choice of $E_{52}$ and $n$. Solutions are found for $E_{52}=0.5,1,2,3$ (see Fig. 6) and values of $n$ between 100 and 400 . In Fig. 7 we find a combination of parameter values, $\epsilon_{\mathrm{B}}=9.0 \times 10^{-4}, \epsilon_{\mathrm{e}}=0.36, E_{52}=1, n=350$ for which the predicted light curve agrees with the data. In this case the logarithmic corrections to the IC spectrum are significant between $0.2-10 \mathrm{keV}$, and they have been added to the power-law approximation. The IC spectrum has been normalized to a peakflux value of $f_{\max }^{\mathrm{IC}}=14 / 45 \times f_{\max } \sigma_{\mathrm{T}} n R$ (Sari \& Esin 2001), where $\sigma_{\mathrm{T}}$ is the Thompson cross section.

In the optical band (Fig. 4, lower panel), the addition of a Galactic-like extinction term (Cardelli et al. 1989, with $R_{V}=$ $3.1)$, with $A\left(V_{\text {int }}\right) \sim 0.15 \mathrm{mag}$ in the GRB site $(z=2.44)$, allows us to explain both the $R$ - and $V$-band observations. A SMC-like environment with $A\left(V_{\text {int }}\right) \sim 0.13$ mag could also be an alternative solution. The implied values of the local $N_{\mathrm{H}}$ are $\sim 0.15 \times 1.79 \times 10^{21} \mathrm{~cm}^{-2}$ and $\sim 0.13 \times 1.6 \times 10^{22} \mathrm{~cm}^{-2}$, both fully compatible with the upper-limit from the X-ray analysis. (For an SMC-like environment, as already noted, this upper-limit should be increased by a factor of $\sim 7$.)

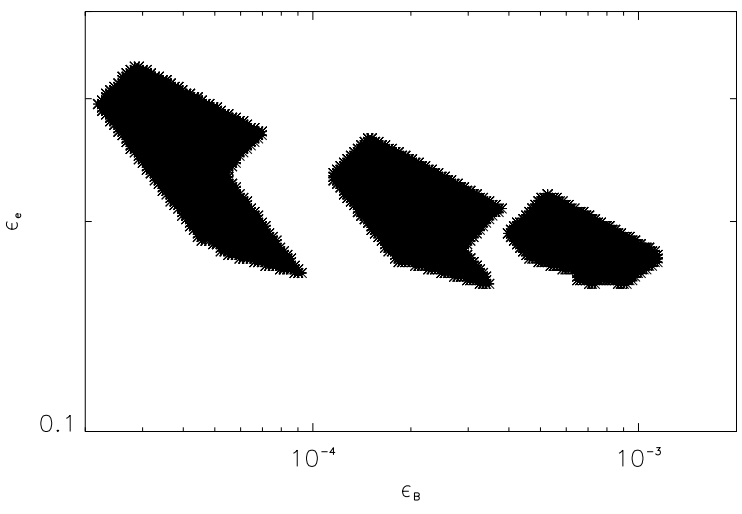

Fig. 5. Numerical test results showing for which values of $\epsilon_{\mathrm{B}}$ and $\epsilon_{\mathrm{e}}$ the conditions expressed by Eqs. (3) to (8) are simultaneously satisfied (and $x>10$ up to $10^{6} \mathrm{~s}$ ). We repeated this search for $E_{52}=0.5,1,2,3,5,10$ combined with $n=10,50,100,150,200,250,300,350,400$. Solutions are found for $E_{52}=3$ and $n=100,150,200$ (shadowed region on the right); $E_{52}=5$ and $n=100,150,200$ (shadowed region in the center); $E_{52}=10$ and $n=100,150,200$ (shadowed region on the left). The three regions represent, for each value of $E_{52}$, the superposition of regions similar to those represented in Fig. 2, each corresponding to one value of $n$ among the ones allowed for the considered value of $E_{52}$. For example, the central region is the superposition of the three regions shown in the upper-left, upper-right, and lower-left panels of Fig. 2.
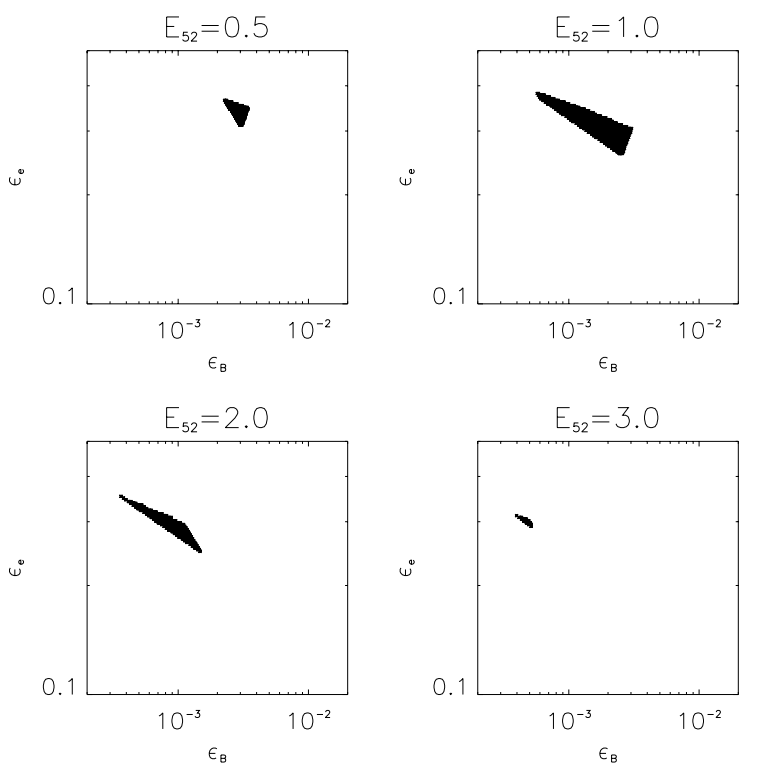

Fig. 6. The shadowed regions are the parts of the $\epsilon_{\mathrm{B}}-\epsilon_{\mathrm{e}}$ plane where the required conditions (see text) are simultaneously satisfied (and $x>10$ up to $10^{6} \mathrm{~s}$ ). Solutions are found for $E_{52}=0.5$ and $n=350,400$ (upper-left panel), $E_{52}=1$ and $n=200,250,300,350,400$ (upperright panel), $E_{52}=2$ and $n=150,200,250,300,350$ (lower-left panel), and $E_{52}=3$ and $n=150,200$ (lower-right panel).

We can finally focus on the spectral predictions of the model we are proposing to explain the XRF 050406 late-time flattening. In a synchrotron-plus-IC scenario, since the light curve flattening is associated with $v_{\mathrm{m}}^{\mathrm{IC}}$ crossing the $\mathrm{X}$-ray band, the spectral index should vary between the values of $-1 / 3$ (for $t \lesssim 10^{5} \mathrm{~s}$ the $\mathrm{X}$-ray band is below $\left.v_{\mathrm{c}}^{\mathrm{IC}}\right)$ and $\sim(p-1) / 2$ for the first solution proposed here (Fig. 3) and between 0.5 (for $2 \times 10^{4} \mathrm{~s} \lesssim t \lesssim 2 \times 10^{5} \mathrm{~s}$ the X-ray band is between $v_{\mathrm{c}}^{\mathrm{IC}}$ and $v_{\mathrm{m}}^{\mathrm{IC}}$ ) and $p / 2$ (plus logarithmic term corrections) for the second solution (Fig. 7). The predicted mean spectral indices between $2.0 \times 10^{4} \mathrm{~s}$ and $10^{6} \mathrm{~s}$ 


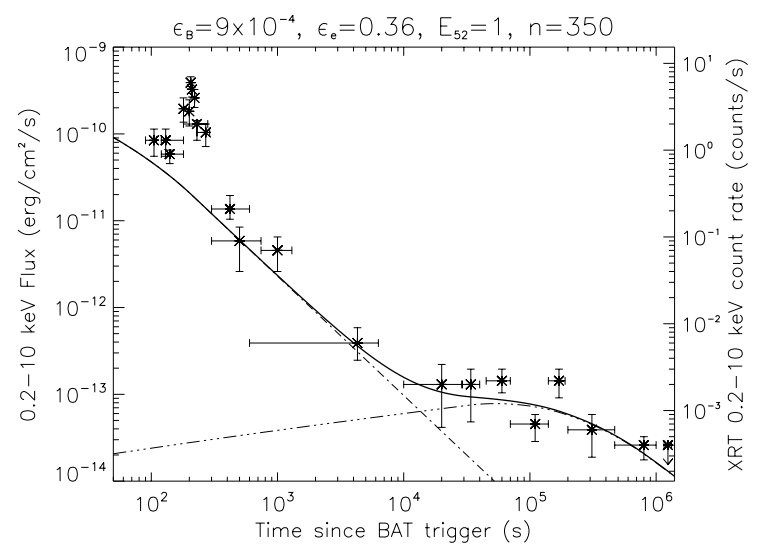

Fig. 7. Synchrotron plus IC model predictions for the $0.2-10 \mathrm{keV}$ light curve with $\epsilon_{\mathrm{B}}=9 \times 10^{-4}, \epsilon_{\mathrm{e}}=0.36, E_{52}=1, n=350, p=2.5$, compared with the observed data (crosses). The dash-dotted line is the contribution from the synchrotron component while the dash-dot-dotdotted line the one from IC emission. The solid line is the resulting total flux.

are $\langle\beta\rangle \sim 0.4$ and $\langle\beta\rangle \sim 0.8$ for the first and second solutions, respectively. Comparing those values with the mean spectral index of $\beta=1.1 \pm 0.3$, measured before the flattening (Romano et al. 2006), it is evident that a hardening should be observed at late times.

For XRF 050406, about 60 source counts were collected between $\sim 2.0 \times 10^{4} \mathrm{~s}$ and $\sim 10^{6} \mathrm{~s}$, so that a detailed spectral analysis cannot be performed. A ratio of $1.8 \pm 0.5$ between hard $(H$ : $0.2-1.0 \mathrm{keV})$ and soft $(S: 1.0-10 \mathrm{keV})$ photons in the whole interval time is the only information that one can get from this faint source (private communication by P. Romano 2006). Comparing this with $0.5 \lesssim H / S \lesssim 1.0$ obtained by Romano et al. (2006) after the flare (see the lowest panel in Fig. 3 of Romano et al. 2006), the spectrum during the late-time flattening seems to be harder, but no firm conclusion can be reached due to the large errors. Since hardening is a natural expectation in a synchrotronplus-IC scenario, we then investigated on the compatibility of our predictions with the observed $H / S$. Using the XRT PC response $^{2}$ (Grade $0-12$, on-axis counts, infinite extraction region), assuming $N_{\mathrm{H}}=2.8 \times 10^{20} \mathrm{~cm}^{-2}$, and normalizing the spectrum so as to have about 60 counts in total, we computed a rough estimate of the expected $\mathrm{H} / \mathrm{S}$ ratio for a spectrum with $\langle\beta\rangle \sim 0.4$ and $\langle\beta\rangle \sim 0.8$, obtaining $H / S=2.4 \pm 0.7$ and $H / S=1.5 \pm 0.4$ for the two spectra ${ }^{3}$; those values of $H / S$ are both compatible with the observed value of $1.8 \pm 0.5$ within the errors. Although a conclusive statement is not allowed, encouraging indications in favor of the present model as a mechanism for the late-time flattening arise globally.

We thus conclude that future observations of brighter sources, possibly allowing performance of time-resolved spectroscopy during the late-time flattening, will be a key to confirming or rejecting the scenario we are suggesting and its potential extension as a general explanation for this kind of late-time behavior in GRB X-ray light curves.

\footnotetext{
${ }^{2}$ http://heasarc.nasa.gov/Tools/w3pimms.html

3 To check for consistency, we also computed the expected hardness ratio for $\beta=1.1$ and 100 source counts. We obtained $H / S=0.98 \pm 0.19$, consistent with the values of $H / S$ found by Romano et al. (2006) after the flare.
}

\section{Conclusions}

We discussed the XRF 050406 afterglow in the context of the standard fireball model. Within a synchrotron-plus-IC scenario, we tested whether the late-time flattening observed in the X-ray light curve can be explained by the appearance of an IC component. We found that setting $\epsilon_{\mathrm{B}}=1.9 \times 10^{-4}, \epsilon_{\mathrm{e}}=0.25, E_{52}=5$, and $n=100$ can explain the X-ray observations. Considering the optical data as well, we noted that the early optical-to-X-ray spectral index appears rather flat and requires additional extinction to recover consistency with the data. We also proposed a second solution with $\epsilon_{\mathrm{B}}=9 \times 10^{-4}, \epsilon_{\mathrm{e}}=0.36, E_{52}=1, n=350$, where the optical-to-X-ray normalization problem is solved requiring that at $t<210 \mathrm{~s}$ some contribution to the X-ray emission comes from the rising part of the flare. Using a Galactic- or SMC-like extinction curve, the $N_{\mathrm{H}}$ required by the optical data is consistent with the upper-limit found in the X-ray analysis.

Acknowledgements. L. Piro acknowledges the support of the EU through the EU FPC5 RTN "Gamma-ray burst, an enigma and a tool"; A. Corsi acknowledges the support of an INFN grant. We thank Pat Romano for performing the spectral analysis of the late-time data and providing the information necessary to test an important aspect of the present work; A. Corsi would also like to thank Pat Romano for interesting general discussions, Giovanni Montani for important comments and suggestions, and Patricia Schady for useful discussions of the optical data.

\section{References}

Barthelmy, S. D., Barbier, L. M., Cummings, J. R., et al. 2005, Space Sci. Rev., 120, 143

Berger, E., Kulkarni, S. R., Fox, D. B., et al. 2005a, ApJ, 634, 501

Berger, E., Oemler, G., \& Gladders, M. 2005b, GRB Coordinates Network, 3185 Burrows, D. N., Hill, J. E., Nousek, J. A., et al. 2005a, Space Sci. Rev., 120, 165 Burrows, D. N., Romano, P., Falcone, A., et al. 2005b, Science, 309, 1833

Capalbi, M., Perri, M., Romano, P., et al. 2005, GRB Coordinates Network, 3184 Cardelli, J. A., Clayton, G. C., \& Mathis, J. S. 1989, ApJ, 345, 245

Chevalier, R. A., \& Li, Z.-Y. 1999, ApJ, 520, L29

Chincarini, G., Moretti, A., Romano, P., et al. 2005, ApJ, submitted [arXiv:astro-ph/0506453]

Corsi, A., Piro, L., Kuulkers, E., et al. 2005, A\&A, 438, 829

Cusomano, G., Kennea, J., Burrows, D. N., et al. 2005, GRB Coordinates Network, 3181

Dickey, J. M., \& Lockman, F. J. 1990, ARA\&A, 28, 215

Fan, Y. Z., \& Wei, D. M. 2005, MNRAS, 364, L42

Galli, A., \& Piro, L. 2006, A\&A, 455, 413

Gehrels, N., Chincarini, G., Giommi, P., et al. 2004, ApJ, 611, 1005

Heise, J., in't Zand, J., Kippen, R. M., \& Woods, P. M. 2001, Gamma-Ray Bursts in the Afterglow Era, 16

Krimm, H., Barbier, L., Barthelmy, S., et al. 2005, GRB Coordinates Network, 3183

Landsman, W., Hunsberger, S., Breeveld, A., et al. 2005, GRB Coordinates Network, 3182

Nousek, J. A., Kouvelioutou, C., Grupe, D., et al. 2006, ApJ, 642, 389

Panaitescu, A., \& Kumar, P. 2000, ApJ, 543, 66

Parsons, A., Barthelmy, S., Cummings, J., et al. 2005, GRB Coordinates Network, 3180

Pei, Y. C. 1992, ApJ, 395, 130

Piro, L., De Pasquale, M., Soffitta, P., et al. 2005, ApJ, 623, 314

Rol, E., Schady, P., Hunsberger, S., et al. 2005, GRB Coordinates Network, 3186 Romano, P., Moretti, A., Banat, P. L., et al. 2006, A\&A, 450, 59

Roming, P. W. A., Kennedy, T. E., Mason, K. O., et al. 2005, Space Sci. Rev., 120,95

Sari, R., \& Esin, A. A. 2001, ApJ, 548, 787

Sari, R., Piran, T., \& Narayan, R. 1998, ApJ, 497, L17

Schady, P., Mason, K. O., Osborne, J. P., et al. 2006, ApJ, 643, 276

Schlegel, D. J., Finkbeiner, D. P., \& Davis, M. 1998, ApJ, 500, 525

Stratta, G., Fiore, F., Antonelli, L. A., et al. 2004, ApJ, 608, 846

Tagliaferri, G., Goad, M., Chincarini, G., et al. 2005, Nature, 436, 985

Wei, D. M., \& Lu, T. 1998, ApJ, 505, 252

Wei, D. M., \& Lu, T. 2000, A\&A, 360, L13

Wu, X. F., Dai, Z. G., Wang, X. Y., et al. 2005, ApJ, submitted

[arXiv:astro-ph/0512555] 